$\sqrt{15}$

Desafío Diagnóstico

\title{
Aporte diagnóstico de la ecocardiografía modo $M$ de la válvula pulmonar
}

Julián Vega, Luigi Gabrielli, Paul McNab, Samuel Córdova.

División de Enfermedades Cardiovasculares. Escuela de Medicina. Pontificia Universidad Católica de Chile.

Correspondencia:

Dr. Julián Vega Adauy

www.ecocardio.cl

julianvega@gmail.com 
Mujer de 55 años, sin comorbilidades conocidas, consulta por disnea de moderados esfuerzos de 1 año de evolución, el examen físico no objetiva elementos de insuficiencia cardíaca. La ecocardiografía Doppler transtorácica (ETT) muestra leve dilatación bi-auricular, tamaño y función bi-ventricular normal y ausencia de insuficiencia tricuspídea. En el modo M de la válvula pulmonar, se identifican dos hallazgos que orientan el diagnóstico etiológico de la disnea y que se presentan como desafío diagnóstico al lector (Figura 1).

\section{Figura 1. Modo M de la válvula pulmonar}

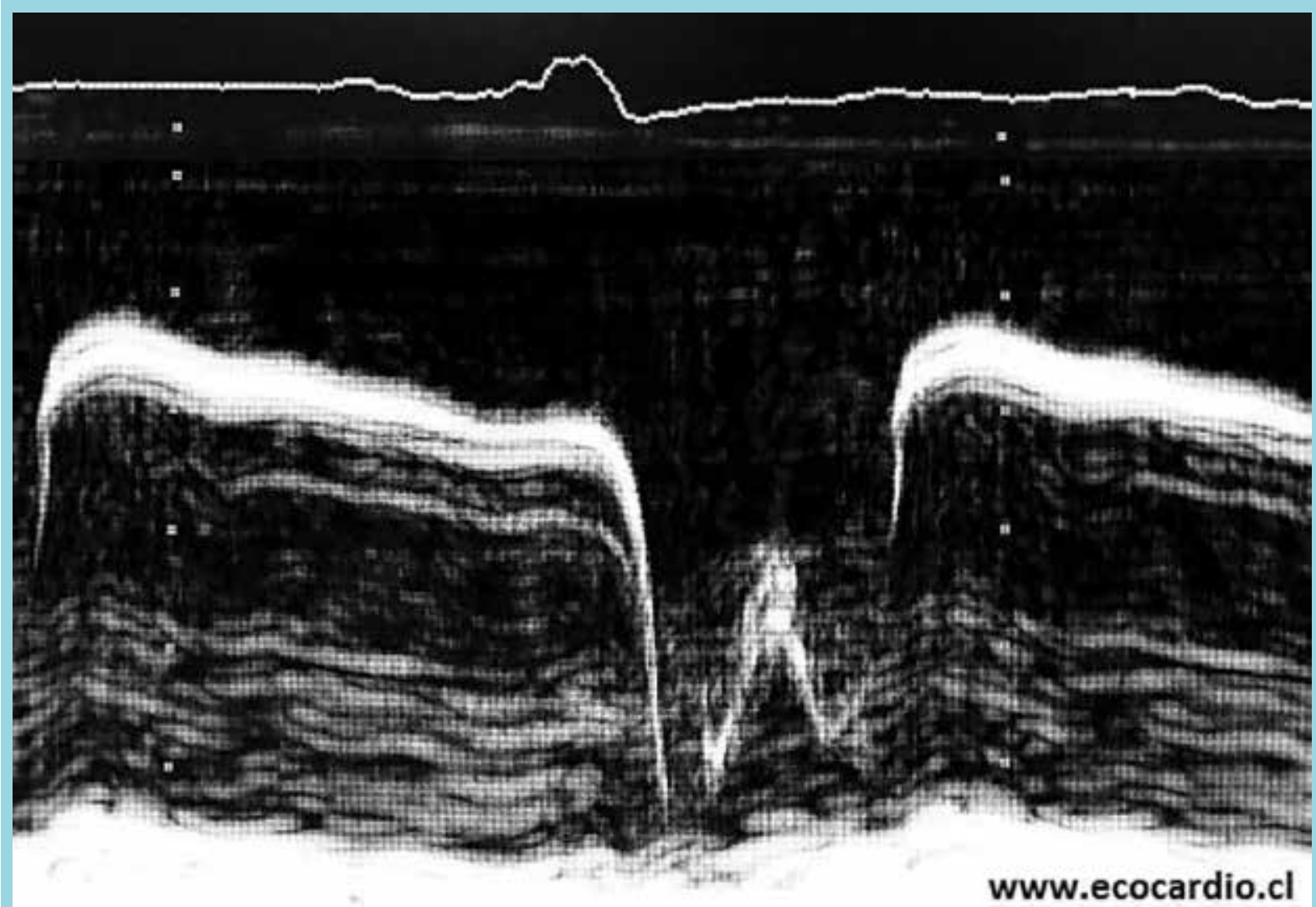




\section{Discusión:}

La estimación no invasiva de la presión de arteria pulmonar (PAP) se realiza mediante ecocardiografía Doppler, midiendo la velocidad máxima $(\mathrm{m} / \mathrm{s})$ de la insuficiencia tricuspídea (IT), que se incorpora en la ecuación simplificada de Bernoulli [gradiente máximo $=4 \mathrm{x}$ (velocidad máxima) ${ }^{2}$; sumando a esto la presión de la aurícula derecha se obtiene la presión sistólica de la arteria pulmonar (PSAP). Sin embargo, en ausencia de insuficiencia tricuspídea no es posible estimar la PSAP, debiendo recurrir a otros elementos ecocardiográficos para estimar la PAP, como por ejemplo, la evaluación de la válvula pulmonar mediante Doppler o modo M.

La Figura 1 es un trazado en modo $\mathrm{M}$ de la válvula pulmonar., En la parte superior de la imagen se observa el registro de ECG y en la parte inferior se observa el modo $\mathrm{M}$ de uno de los velos de la válvula pulmonar (habitualmente el velo posterior), velo que durante la diástole queda representado por una línea continua (válvula cerrada) y durante la

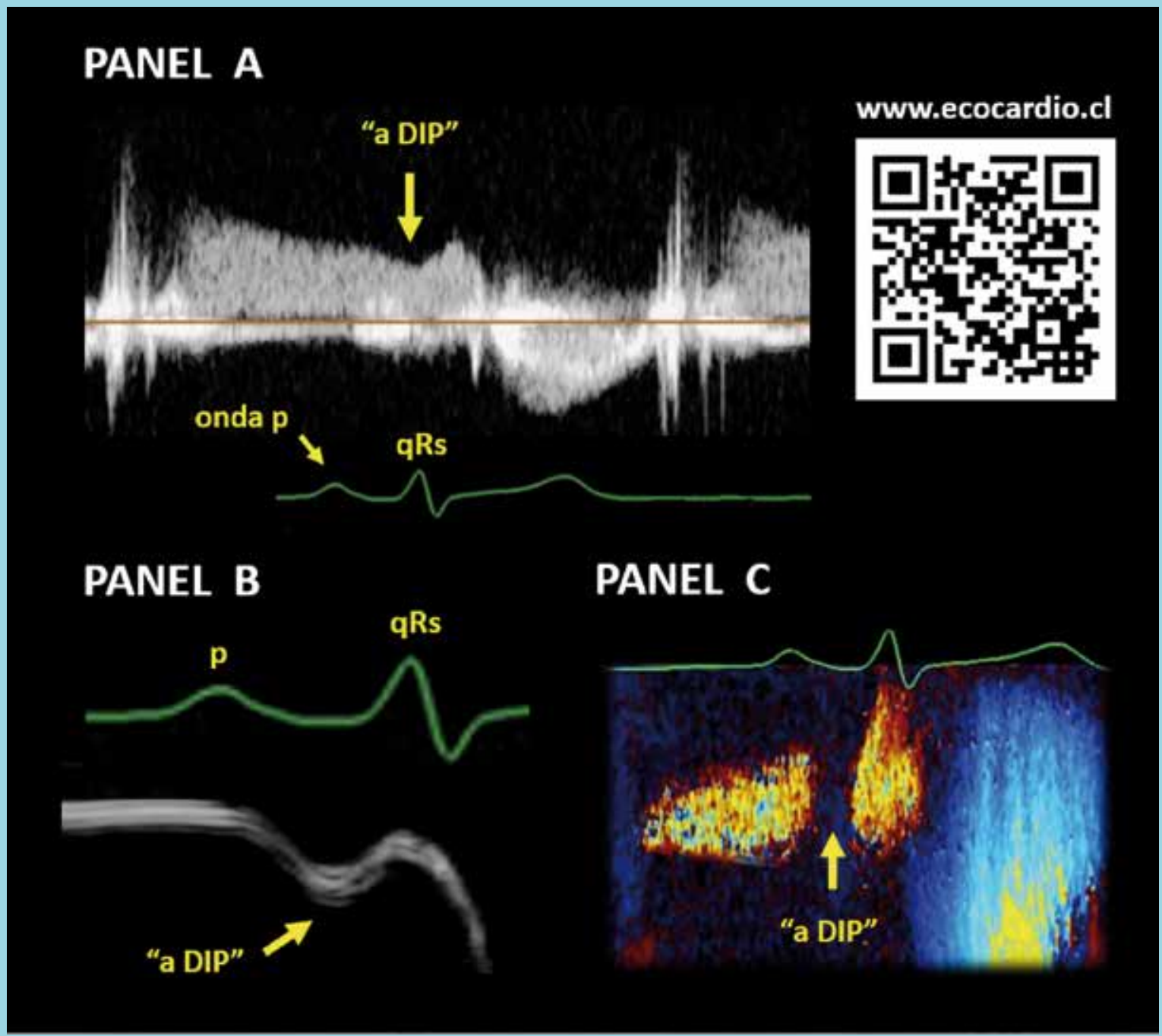

Panel A: Doppler continuo a través de la válvula pulmonar, se observa en la señal de insuficiencia pulmonar una escotadura o muesca (“a DIP”) producida por la contracción auricular derecha.

Panel B: Modo M de la válvula pulmonar que evidencia la muesca y su relación con la onda A del ECG.

Panel C: Modo M color de la válvula pulmonar que evidencia disminución de la insuficiencia pulmonar producto del "DIP” auricular normal. Más información escaneando el código QR (esquina superior derecha) 


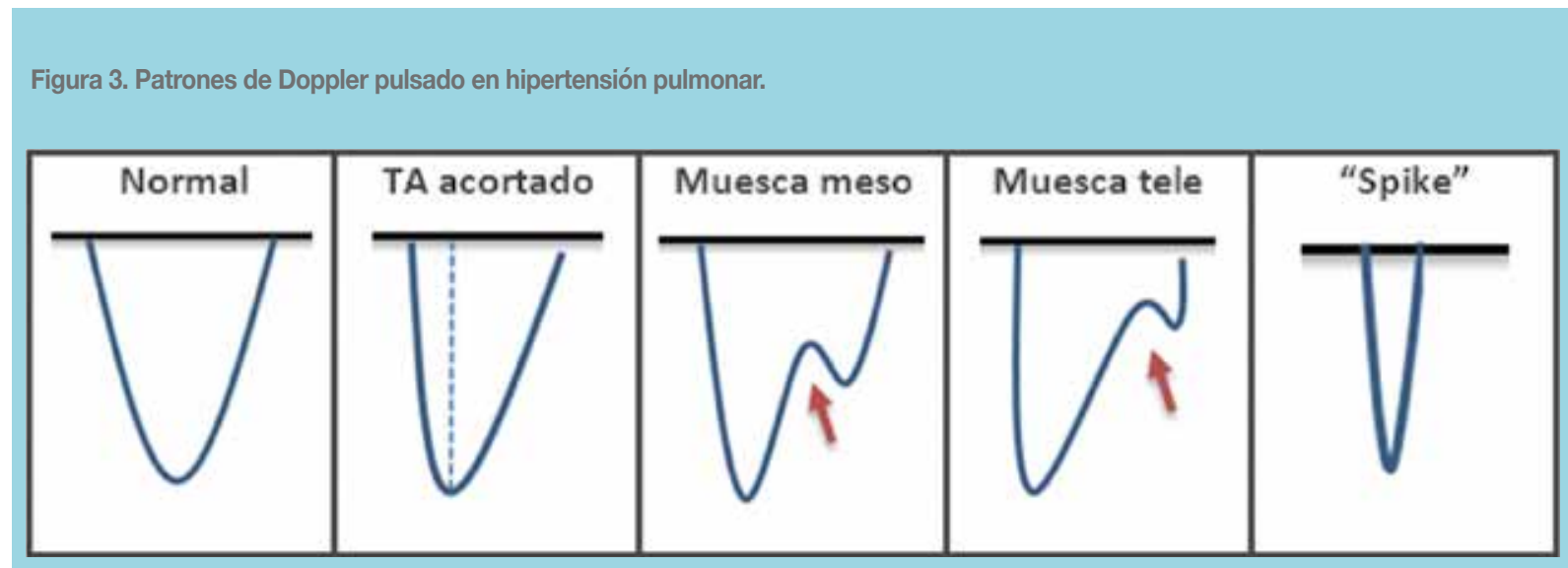

Patrón de izquierda a derecha; 1. Normal, 2. Tiempo de aceleración acortado (triangular), 3. Muesca meso sistólica, 4. Muesca tele sistólica. 5. Patrón triangular pequeño "spike" (asociado a bajo gasto).

sístole evidencia un movimiento que inicialmente se aleja del transductor que refleja la apertura valvular. En dicho trazado destacan dos hallazgos patológicos que orientan a la etiología de la disnea y que se detallan a continuación:

\section{Ausencia de onda "a"}

En ritmo sinusal y posterior a la presencia de la onda A del ECG, en respuesta a la contracción auricular derecha se genera una muesca o "DIP" diastólico en la válvula pulmonar (onda "a"), observable en modo M, Doppler pulsado y continuo (Figura 2, panel A). Cuando existe hipertensión pulmonar (HTP), la contracción auricular derecha enfrentada a una mayor postcarga no logra generar dicha muesca sobre la válvula o el efecto generado es muy pequeño (muesca $<2 \mathrm{~mm}$ ), relacionándose así la ausencia de la onda "a" en el modo M con una presión media de arteria pulmonar $>40 \mathrm{mmHg}^{1}$.

\section{Muesca meso-sistólica.}

En condiciones normales la válvula pulmonar presenta una apertura completa y simétrica durante todo el sístole. En presencia de HTP muchos pacientes presentan un cierre parcial y transitorio de la válvula pulmonar durante el sístole (meso o tele sístole), generando una "muesca" sistólica que se observa tanto en el modo $\mathrm{M}$ como en el Doppler (pulsado y continuo) del tracto de salida del ventrículo derecho y constituye uno de los patrones de Doppler pulsado reportados en $\mathrm{HTP}^{2}$, que se resumen en la Figura 3.

\section{Fisiopatología de la muesca sistólica}

La contracción de las cámaras cardíacas genera ondas que viajan en dirección anterógrada y que al encontrar obstáculos en su camino como, por ejemplo, la bifurcación de la arteria pulmonar, producen ondas reflejas o de rebote que viajan en dirección retrógrada. Cuando la resistencia vascular pulmonar se encuentra elevada, la mayor impedancia al flujo produce que este reflejo o rebote sea de mayor magnitud y, además, ocurra de forma prematura en sístole. Esta onda refleja anticipada encuentra a la válvula pulmonar aún abierta hacia meso o tele sístole, generando sobre ella una muesca o "notch". Se ha propuesto, además, que un reflejo más precoz produce un notch en meso-sístole y se relaciona a sitios de obstrucción más proximales en la vasculatura pulmonar como, por ejemplo, la enfermedad tromboembólica crónica (ETEC) y un reflejo más tardío en sístole produce un notch tele-sistólico y se relaciona con enfermedad vascular pulmonar más distal, como ocurre en la hipertensión arterial pulmonar (primaria).

Asimismo, existe correlación entre la presencia de dicha muesca con RVP> 3 U Woods. No obstante, debe considerarse que $40 \%$ de los pacientes con HTP pueden no presentar muesca en la válvula pulmonar, debido a que existen otras variables, sin relación al grado de HTP, que determinan su aparición, tales como: el tiempo de evolución de la HTP, la causa de HTP (menos frecuente en HTP causada por falla del corazón izquierdo), el bajo gasto cardíaco y la dilatación de la arteria pulmonar. 


\section{Utilidad}

La sensibilidad diagnóstica de la muesca sistólica para diagnosticar HTP varía entre $60-90 \%{ }^{3}$, con una especificidad cercana al $100 \%$. Vale la pena mencionar que la especificidad no es $100 \%$, ha sido reportado en ausencia de HTP en el infrecuente caso de dilatación idiopática de la arteria pulmonar.

Los hallazgos anteriormente descritos tienen utilidad en los siguientes escenarios:

- En presencia de señal de IT que permite estimar la PSAP, la presencia de una muesca respalda la presencia de HTP. En el caso de medir una PSAP normal, su presencia sugiere que se está subestimando la real PAP (subregistro de la velocidad máxima de la IT, ecualización de gradientes entre AD-VD como en el caso de IT severa).
- En ausencia de señal de IT para el cálculo de la PSAP, es en un signo indirecto de HTP. Otros hallazgos ecocardiográficos útiles en ausencia de IT son; la presencia de insuficiencia pulmonar que mediante el Doppler pulsado permite el cálculo de la presión media y diastólica de la arteria pulmonar, el acortamiento del tiempo de aceleración de la válvula pulmonar ( $<100 \mathrm{~ms})$ y la sobrecarga de presión y el remodelado de las cavidades derechas.

Finalmente, debe considerarse que el cálculo de la PSAP tiene limitaciones, por tanto el valor obtenido debe ser interpretado en conjunto con otros parámetros de HTP y del corazón derecho, como la presencia de una muesca sistólica que apoya fuertemente el diagnóstico de HTP. Por último, frente a inconsistencias debe tenerse presente que el estándar diagnóstico es el cateterismo cardíaco derecho. 
1. NANDA NC, GRAMIAK R, ROBINSON T, SHAH P. Echocardiographic Evaluation of Pulmonary Hypertension. Circulation. 1974;50:575-581.

2. ARKLES JS, OPOTOWSKY AR, OJEDA J, et al. Shape of the Right Ventricular Doppler Envelope Predicts Hemodynamics and Right Heart Function in Pulmonary Hypertension. Am J Respir Crit Care Med. 2011;183:268-276.
3. KITABATAKE A, INOUE M, ASAO M, et al. Noninvasive evaluation of pulmonary hypertension by a pulsed Doppler technique. Circulation. 1983;68:302-309.

4. BAUMAN W, WANN LS, CHILDRESS R, WEYMAN AE, FEIGENBAUM H, DILLON J. MID systolic notching of the pulmonary valve in the absence of pulmonary hypertension. Am J Cardiol. 1979;43:1049-1052. 